\title{
Fetal Tachycardia in the Delivery Room: Fetal Distress, Supraventricular Tachycardia, or Both?
}

\author{
1 Paediatric and Neonatal Services, North Shore Hospital, Auckland, \\ New Zealand \\ 2 Department of Paediatrics, Faculty of Medical and Health Sciences, \\ The University of Auckland, Auckland, New Zealand \\ ${ }^{3}$ North Shore Hospital, Auckland, New Zealand \\ ${ }^{4}$ Department of Obstetrics and Gynaecology, Faculty of Medical and \\ Health Sciences, The University of Auckland, Auckland, New Zealand \\ 5 The Childrens Hospital at Westmead, Sydney Children's Hospital \\ Network, Sydney, NSW Australia
}

Satvinder Singh Bhatia, BM(Soton), FRACP(Paeds) ${ }^{1,2}$ Wendy H. Burgess, BHB/MB ChB, FRANZOG ${ }^{3,4}$ Jonathan R. Skinner, MB ChB DCH MRCP(UK) FRACP FCANZ FHRS MD ${ }^{5}$

\begin{abstract}
Address for correspondence Satvinder Singh Bhatia, BM(Soton), FRACP(Paeds), North Shore Hospital, Private Bag 93-503, Takapuna, Auckland 0740, New Zealand

(e-mail: Satvinder.Bhatia@waitematadhb.govt.nz).
\end{abstract}

Am J Perinatol Rep 2020;10:e380-e385.

\begin{abstract}
Background Supraventricular tachycardia (SVT) is seldom considered a cause for fetal tachycardia; commoner etiologies including maternal fever and fetal distress are usually envisaged. Fetal arrhythmia can be missed as a diagnosis, potentially leading to suboptimal management.

Cases Three cases are described where detection of fetal tachycardia $>200$ beats per minute (bpm) at 36,40 , and 38 weeks gestation resulted in emergency cesarean section for presumed fetal distress. Retrospective review of the cardiotocograph in two cases revealed baseline heart rates 120 to $160 \mathrm{bpm}$, with loss of trace associated with auscultated rates over $200 \mathrm{bpm}$. The diagnosis of SVT was not initially considered and made later when the infants required cardioversion at the age of 3 weeks, 2 days, and 8 days, respectively. The 36 -week infant required noninvasive ventilation for prematurity.

Conclusion SVT should be actively considered in the differential diagnosis of fetal tachycardia. Unrecognized fetal SVT may result in avoidable caesarean for suspected

Keywords

- fetal distress

- fetal arrhythmia

- fetal SVT

- neonatal SVT fetal distress, with potential prematurity-related problems. The cardiotocograph can be helpful if showing contact loss associated with rapid heart rate auscultation. The antenatal detection of fetal SVT is important as it can allow anticipation and prevention of neonatal SVT, which is potentially life-threatening if not detected and treated promptly.
\end{abstract}

When supraventricular tachycardia (SVT) in the fetus is recognized antenatally, the management requires balancing risks of continuing pregnancy versus delivery, while considering gestation, presence of hydrops and cardiac failure. ${ }^{1}$ The treatment is well described, ${ }^{2-5}$ typically involving the use of antiarrhythmics being given to the mother, and there are some data on postnatal outcomes. ${ }^{6,7}$

received

July 26, 2020 accepted after revision August 21, 2020
DOI https://doi.org/

10.1055/s-0040-1718900. ISSN 2157-6998.
However, there is another scenario that is not welldescribed: that of the misdiagnosis of fetal SVT as sinus tachycardia due to fetal distress during the peripartum period. In practice, the obstetrician or midwife is more likely to think of commoner etiologies for fetal tachycardia such as maternal fever, dehydration, thyroid disorders, and fetal distress. There is a risk that SVT is not considered in the
Copyright $\odot 2020$ by Thieme Medical Publishers, Inc., 333 Seventh Avenue, New York, NY 10001, USA. Tel: +1(212) 760-0888
License terms

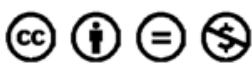


differential diagnosis, as illustrated here. This can potentially result in suboptimal management including an unnecessary emergency cesarean section, as could be argued in case 2 .

Characteristics that distinguish SVT from sinus tachycardia include a much faster heart rate, typically well over 200 beats per minute (bpm), with abrupt onset and offset. ${ }^{2}$

\section{Case 1}

A 27-year-old woman, primigravida at 36 weeks gestation, presented to hospital with no fetal movements for 48 hours. Fetal heart rate (FHR) was $>200 \mathrm{bpm}$ on cardiotocograph (CTG). Fetal ultrasound scan (USS) demonstrated minimal tone and confirmed FHR $>200 \mathrm{bpm}$. Emergency cesarean section was performed. The baby was initially well but soon developed respiratory distress needing continuous positive airway pressure support and 5 days admission in the neonatal unit. The peak heart rate recorded was $168 \mathrm{bpm}$. A diagnosis of SVT was not considered and no electrocardiogram (ECG) was obtained.

At the age of 3 weeks, the infant presented to the general practitioner with 2 days poor feeding, vomiting and irritability, and looked unwell with cool peripheries, heart rate $280 \mathrm{bpm}$, and was referred to hospital with suspected sepsis. On presentation to emergency, the infant was very unwell with heart rate $280 \mathrm{bpm}$. There was a narrow complex tachycardia on ECG, which was refractory to cold water immersion. The infant received intravenous fluid and multiple adenosine doses, but became increasingly drowsy and mottled. A $15 \mathrm{~J}(\mathrm{~J})$ direct current synchronized shock led to reversion to sinus rhythm. After stabilization and transfer to the tertiary hospital for Cardiology and Pediatric Intensive Care the ECG in sinus rhythm showed delta waves consistent with Wolff-Parkinson-White (WPW) syndrome.

Subsequently progress was characterized by recurrent SVT episodes requiring multiple changes in medication prophylaxis using flecainide, atenolol, and sotalol. At the age of 4 years, radiofrequency ablation of a left sided accessory pathway was performed, and at the age of 8 years, he remains off medication with no SVT.

\section{Case 2}

A 36-year-old woman, gravida 4 parity 2, with uncomplicated pregnancy at term, presented in spontaneous labor. CTG showed FHR 120 to $160 \mathrm{bpm}$ with periods of loss of trace simultaneous to auscultation FHR 220 to $240 \mathrm{bpm}$ (-Fig. 1). Considering that this represented fetal distress, an emergency cesarean section was performed. At birth, the infant was well with heart rate $124 \mathrm{bpm}$. SVT was not considered.

The following day, the infant remained well, but the heart rate was too rapid to count manually. An ECG showed heart rate $266 \mathrm{bpm}$ and narrow QRS complexes consistent with SVT (-Fig. 2 upper panel). On repeat auscultation, the heart rate had spontaneously changed to a slower rate and ECG shows sinus rhythm without delta waves ( - Fig. 2 lower panel). Echocardiography was normal.
On day 3, recurrent SVTs were reverted with adenosine. The infant was then successfully treated with flecainide prophylaxis until age 2 years. She was diagnosed as having SVT without delta waves and remained off medication without SVT at the age of 8 years.

\section{Case 3}

A 30 -year-old woman, gravida 2 parity 0 , with uncomplicated pregnancy at 38 weeks not in labor, presented with a 1day history of reduced fetal movements. The CTG showed FHR $150 \mathrm{bpm}$ with poor variability and periods of loss of trace, during which the midwife auscultates a very fast heart rate (-Fig. 3). Portable USS detected FHR $220 \mathrm{bpm}$ and low liquor. Emergency cesarean section was performed for presumed fetal distress.

The infant was born in poor condition, hypotonic, cyanosed, gasping, and then apneic. The heart rate was initially heard briefly but too fast to count and then the heart rate was absent. Resuscitation was performed with positive pressure ventilation using Neopuff in room air, with good response, heart rate $>100 \mathrm{bpm}$, and regular respiration by 4 minutes age. Apgar scores were 2, 7 and 8 at 1, 5, and 10 minutes respectively.

The infant was admitted to the neonatal unit and remained well apart from transient feed intolerance. SVT was considered as contributing to his symptoms. Cardiac exam and ECG were normal apart from bifid $\mathrm{p}$ waves on the ECG. The heart rate ranged 92 to $169 \mathrm{bpm}$ for first 72 hours, and peak heart rate was $191 \mathrm{bpm}$ over 7 days.

Cardiac review was arranged in the tertiary hospital on day 8 . At the start of transfer, the baby was well, but during transport, the nurse noted the heart rate to be intermittently up to $300 \mathrm{bpm}$. On arrival in the cardiac unit, the baby had intermittent apnea, weak pulses, and heart rate was 250 to $300 \mathrm{bpm}$. An ECG was consistent with SVT. Adenosine and cold-water immersion reverted the infant to sinus rhythm, who then remained well. ECG post SVT showed delta waves and short PR interval consistent with WPW syndrome. Propranolol prophylaxis was commenced.

The Holter recording at day 8 was noted to show onset of delta wave complexes ( - Fig. 4 ).

The baby was well at 1 month follow-up. Further followup information is unavailable as the family moved overseas.

\section{Conclusion}

This case series describes neonatal SVT first being recognized as such at 3 weeks, 2 days, and 8 days, where retrospective review reveals that the SVT caused perinatal tachycardia, without being recognized as the underlying cause. These cases highlight the importance of recognizing SVT as a potential cause of fetal tachycardia as this diagnosis will be easily missed if it is not even considered. The fetuses did not undergo fetal echocardiography when fetal tachycardia $>200 \mathrm{bpm}$ was detected because the fetus was presumed to have fetal distress and as a result emergency cesarean section was performed. Fetal echocardiography was not available at 


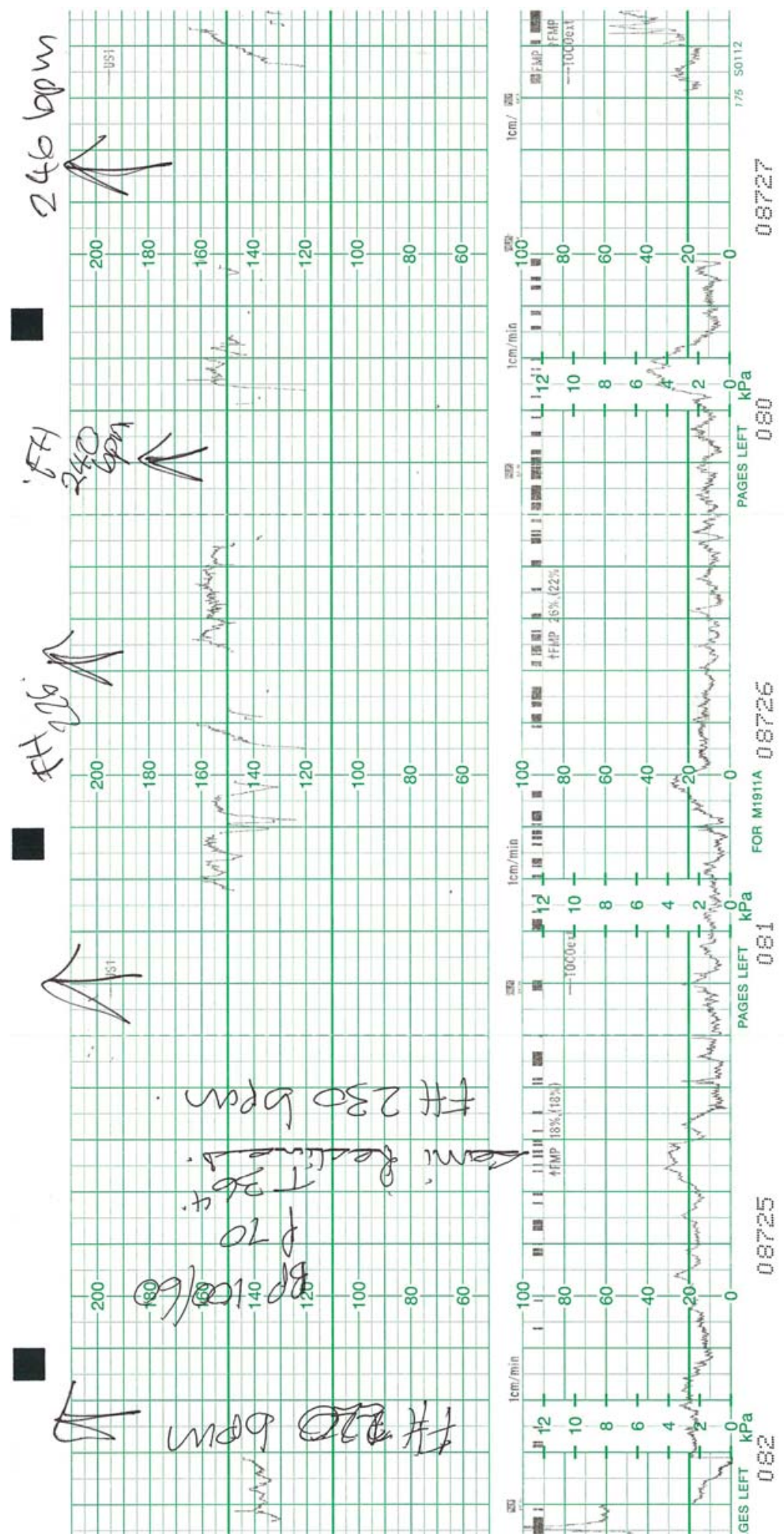

Fig. 1 Cardiotocograph from case 2. In the upper panel, the hand written notes illustrate where the heart rate tracing (vertical scale 50-210 beats per minute, bpm) is lost but the fetal heart rate is documented by auscultation, and found to be 220 to 246 bpm. The lower panel illustrates the pressure of the uterine contractions (vertical scale 0-100 kilopascals, kPa). 


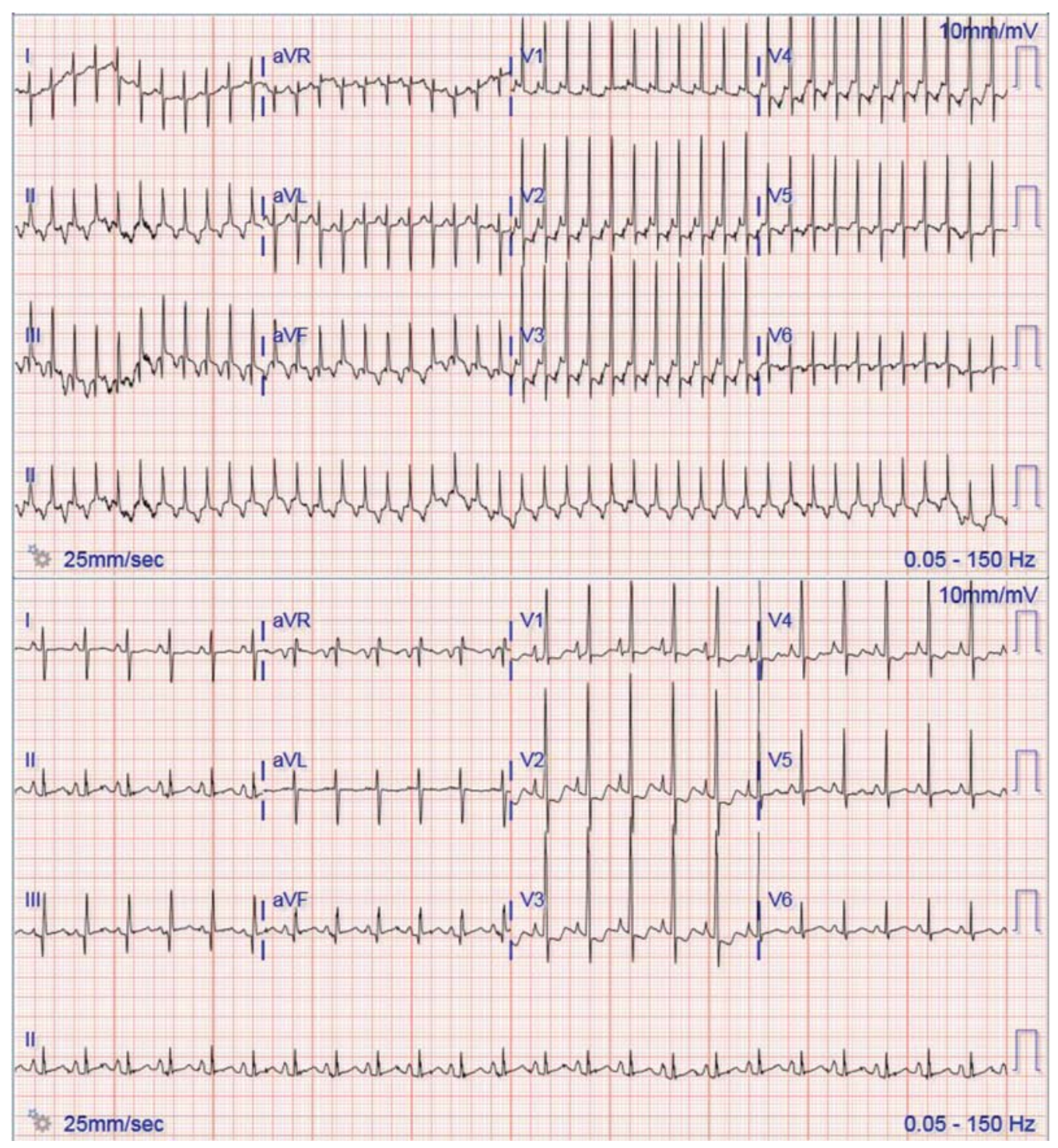

Fig. 2 Twelve lead electrocardiogram from case 2 illustrating supraventricular tachycardia in the upper panel and sinus rhythm in the lower panel after spontaneous resolution. No pre-excitation is evident.

the hospital where the babies were delivered; referral to a center with facilities for fetal echocardiography would have delayed timely delivery of a compromised fetus. In the first and third case, the infant was clearly compromised by the SVT, so that an emergent delivery was, in any case, appropriate. However, the postnatal management should anticipate neonatal SVT, since this can be life-threatening when not recognized early. After delivery of infants who had documented fetal SVT about two thirds will have postnatal arrhythmias, with two-thirds of those presenting within 48 hours. ${ }^{6,7}$ Characteristics that distinguish SVT from sinus tachycardia include a much faster heart rate, typically well over $200 \mathrm{bpm}$, with abrupt onset and offset. ${ }^{2}$ The CTG may lead one to consider this diagnosis; if there is loss of contact of trace at the same time, a rapid heart rate is auscultated or confirmed by fetal ultrasound (-Figs. 1 and $\mathbf{3}$ ).

The next steps in management are determined by the gestation and condition of the fetus as well as advice from specialty services. ${ }^{1}$ Once the diagnosis is suspected, there should be dialogue between the perinatal specialists, including cardiology. Whether delivery needs to be expedited will depend on parameters of fetal well-being other than the heart rate alone. An ECG on the first day or two of life is required for evidence of pre-excitation, with continuous monitoring for 3 to 4 days. Close monitoring is mandated following discharge; prophylactic antiarrhythmics should be considered and many centers will recommend training of parents to use a stethoscope to detect presymptomatic SVT in the first months of life.

Despite being a rare cause of fetal tachycardia, SVT is the most common fetal tachyarrhythmia and is a serious condition that needs to be actively considered in the delivery room. Unrecognized fetal SVT can potentially result in avoidable emergency cesarean for suspected fetal distress, with potential problems associated with a premature delivery. The CTG can be helpful if it shows loss of contact in association with auscultation of rapid heart rate. The antenatal detection of fetal SVT is important since most infants will 
e384 Fetal Tachycardia in the Delivery Room Bhatia et al.

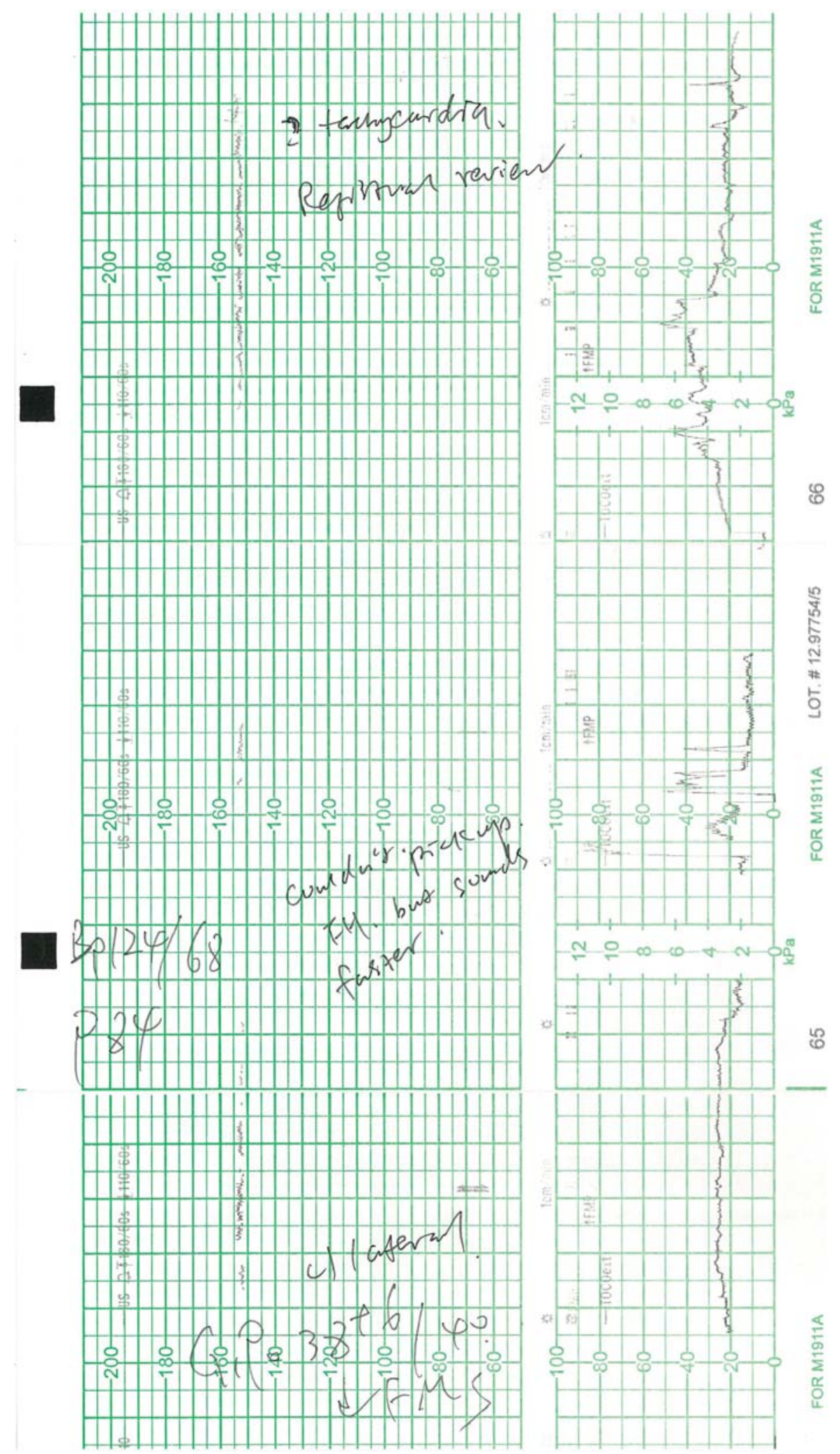

Fig. 3 Cardiotocograph (CTG) from case 3. The upper panel illustrates a poor recording of fetal heart rate $\sim 150$ bpm with reduced variability. There are sections of the CTG where the fetal heart rate is unrecorded that corresponded with fetal heart rate of 220 bpm on portable scan. The lower panel illustrates the pressure of the uterine contractions (vertical scale $0-100 \mathrm{kPa}$ ). 


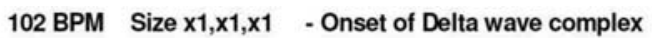

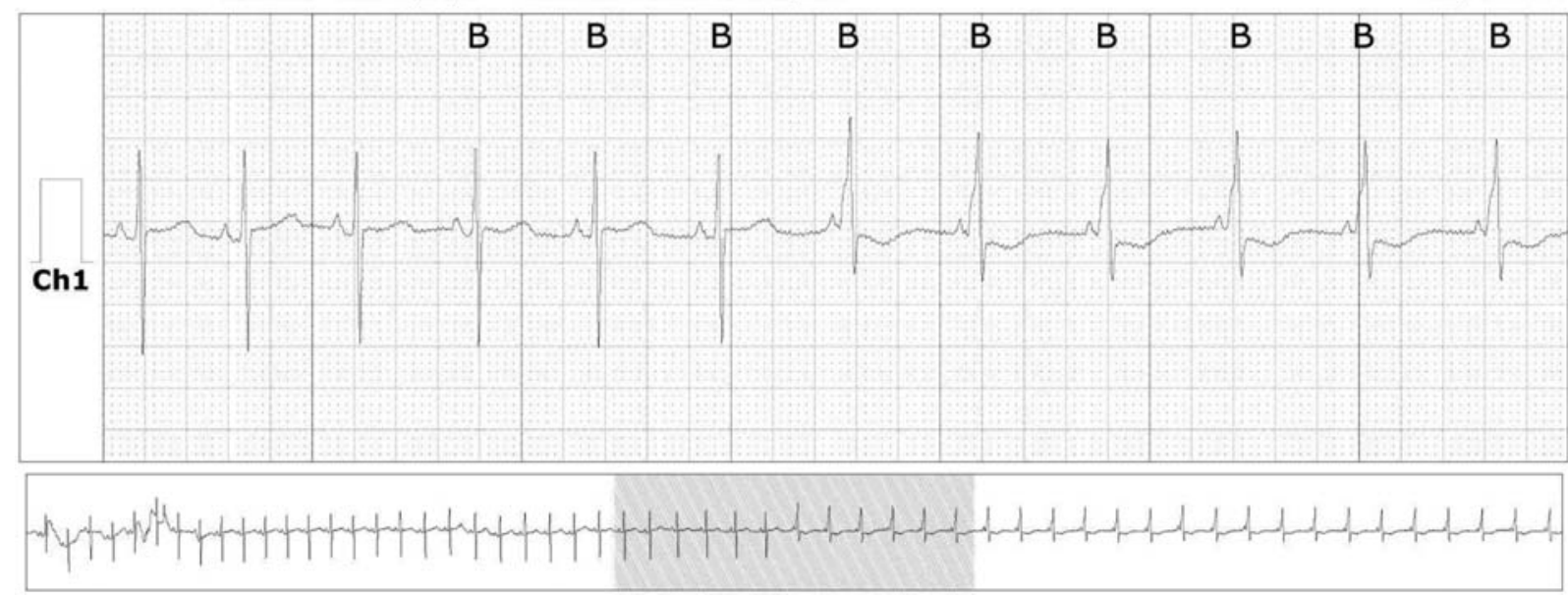

Fig. 4 Holter recording from case 3 on day 8, which illustrates intermittent pre-excitation. Pre-excitation is absent on the first six beats and present on the latter six.

develop SVT postnatally, and this can be life threatening if not detected and treated promptly.

\section{Statement of Ethics}

This research has been registered and has locality authorization with the Waitemata District Health Board, Auckland, New Zealand. Registration number RM13912.

The parents of the infants in case 1 and case 2 were contacted about the research and have given their consent for their infants to be included in this study. An attempt was made to contact the family of the infant in case 3 via their midwife, and they were unable to be contacted due to the family moving overseas.

\section{Conflict of Interest}

The authors have no conflict of interest to disclose.

\section{Acknowledgments}

Ms Jianping Wang RM, Midwife, Maternity Services, North Shore Hospital, Auckland, New Zealand, helped with contacting the families in case 1 and case 3 for whom she was the Lead Maternity Caregiver.
Cardiology Procedures Department, North Shore Hospital, Auckland, New Zealand provided the ECG and Holter images.

\section{References}

1 Simpson LL. Fetal supraventricular tachycardias: diagnosis and management. Semin Perinatol 2000;24(05):360-372

2 Skinner JR, Sharland G. Detection and management of life threatening arrhythmias in the perinatal period. Early Hum Dev 2008; 84(03):161-172

3 Chu PY, Hill KD, Clark RH, Smith PB, Hornik CP. Treatment of supraventricular tachycardia in infants: analysis of a large multicenter database. Early Hum Dev 2015;91(06):345-350

4 Simpson LL, Marx GR, D’Alton ME. Supraventricular tachycardia in the fetus: conservative management in the absence of hemodynamic compromise. J Ultrasound Med 1997;16(07):459-464

5 Gimovsky ML, Nazir M, Hashemi E, Polcaro J. Fetal/neonatal supraventricular tachycardia. J Perinatol 2004;24(03):191-193

6 Moodley S, Sanatani S, Potts JE, Sandor GGS. Postnatal outcome in patients with fetal tachycardia. Pediatr Cardiol 2013;34(01): 81-87

7 Hinkle KA, Peyvandi S, Stiver C, et al. Postnatal outcomes of fetal supraventricular tachycardia: a multicenter study. Pediatr Cardiol 2017;38(07):1317-1323 